\title{
Desempeño en funciones ejecutivas y síntomas comórbidos asociados en niños con Trastorno por déficit de atención con hiperactividad (TDAH)
}

\author{
Performance on Executive Functions and Comorbid Associated \\ Symptoms in Children with Attention Deficit Hyperactivity \\ Disorder (ADHD)
}

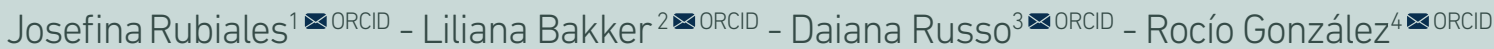 \\ 1,2,3,4 Universidad Nacional de Mar del Plata
}

Argentina

Fecha correspondencia:

Recibido: 23 de diciembre de 2015. Aceptado: julio 19 de 2016.

Forma de citar:

Rubiales, J., Bakker, L., Russo, D., \& González, R. (2016). Desempeño en funciones ejecutivas y síntomas comórbidos asociados en niños con Trastorno por déficit de atención con hiperactividad (TDAH). Rev. CES Psicol., 9(2),99-113.

\section{Open access}

(c) Copyright

Licencia creative commons

Etica de publicaciones

Revisión por pares

Gestión por Open Journal System

ISSN: 2011-3080

DOl: http://dx.doi.org/10.21615/ cesp.9.2.7

Sobre los autores:

1. Dra. en Psicología. Consejo Nacional de Investigaciones Científicas y Técnicas (CONICET). Instituto de Psicología Básica, Aplicada y Tecnología (IPSIBAT). Facultad de Psicología, Universidad Nacional de Mar del Plata.

Comparte

\section{Resumen}

El objetivo del presente estudio fue analizar el desempeño de las funciones ejecutivas en niños con Trastorno por déficit de atención con hiperactividad (TDAH) en relación con síntomas comórbidos asociados, internalizantes y externalizantes. Se evaluaron 45 niños de 8 a 14 años con diagnóstico de TDAH con pruebas de funciones ejecutivas. Los resultados evidenciaron que el grupo de niños con síntomas internalizantes presentaron un desempeño inferior en organización y planificación y en inhibición motora, y el grupo de niños con síntomas externalizantes un desempeño inferior en inhibición motora, en comparación con los niños sin síntomas comórbidos, lo cual indica que las variables analizadas permiten colaborar con el proceso de evaluación diagnóstica del trastorno. Describir el desempeño ejecutivo del trastorno en función de diversas asociaciones comórbidas, posibilita un mejor conocimiento de la entidad y un adecuado diagnóstico diferencial.

Palabras Claves: Comorbilidad, Funciones Ejecutivas, Niños, Trastorno por déficit de atención con hiperactividad, TDAH.

\section{Abstract}

The aim of this study was to analyze the performance of executive functions in children with Attention deficit hyperactivity disorder based on internalizing and externalizing associated comorbid symptoms. 45 children aged 8 to 14 with ADHD were assessed with executive function tests. The results showed that the group of children with internalizing symptoms has underperformed in relation to organization and planning and motor inhibition, and the group of children with externalizing symptoms underperformed motor inhibition compared to children without comorbid symptoms, which would indicate that the analyzed variables allow collaborating with the diagnostic evaluation process of the disorder. Be able to describe the executive function disorder performance in relation to various comorbid associations would enable a better understanding of the entity and an adequate differential diagnosis.

Keywords: ADHD, Attention Deficit Disorder with Hyperactivity, Children, Comorbidity, Cognitive Ability, Executive Functions. 
Pág 100

2. Lic. en Ciencias Biológicas. Esp. En Docencia Universitaria. IPSIBAT. Facultad de Psicología, Universidad Nacional de Mar del Plata.

3. Lic. en Psicología. CONICET. Instituto de Psicología Básica, Aplicada y Tecnología (IPSIBAT). Facultad de Psicología, Universidad Nacional de Mar del Plata.

4. Lic. en Psicología. IPSIBAT. Facultad de Psicología, Universidad Nacional de Mar del Plata.

\section{Introducción}

El Trastorno por déficit de atención e hiperactividad (TDAH) es el trastorno del neurodesarrollo más frecuente iniciado en la infancia (Rodillo, 2015), siendo su sintomatología una de las principales fuentes de derivación de los niños al sistema de salud (Gaitán-Chipatecua \& Rey-Anacona, 2013). El TDAH se define como un patrón persistente de síntomas de inatención, hiperactividad e impulsividad, que es más frecuente y grave que el observado en las personas con un grado de desarrollo similar (Asociación Americana de Psiquiatría [APA], 2013).

Los estudios sobre el TDAH estiman que su prevalencia mundial es de 5,9 a 7,1\% en niños y adolescentes (Willcutt, 2012), siendo más frecuente en niños que en niñas con una relación de tres a uno (Valdizán, Mercado, \& Mercado-Undanivia, 2007). Los hallazgos en cuanto a su etiología son consistentes con una hipótesis multicausal (Catelan-Mainardes, 2010) que da cuenta de una combinación de factores genéticos, biológicos y ambientales (Bakker \& Rubiales, 2010; Labos, Slachevsky, Fuentes, \& Manes, 2008).

El diagnóstico del trastorno resulta un proceso complejo (De la Peña, Palacio, \& Barragán, 2010), ya que hasta el momento no se dispone de pruebas neurobiológicas específicas, ni de marcadores fisiológicos o estudios de neuroimágenes que permitan establecerlo con certeza (Catelan-Mainardes, 2010). Por lo tanto, el diagnóstico se establece por medio del cumplimiento de criterios clínicos específicos (Álvarez. 2006), a partir de los cuales se pueden distinguir tres presentaciones clínicas, de acuerdo al predominio de los síntomas principales: inatento, hiperactivo-impulsivo y combinado o mixto. Dichos síntomas deben presentarse antes de los 12 años de edad, en más de un contexto (casa y escuela) e interferir significativamente en la vida diaria del paciente (APA, 2013).

La literatura científica actual sobre TDAH coincide en señalar que el déficit primario está asociado a una disfunción ejecutiva (Barkley, 2011; Fischer, Barkley, Smallish, \& Fletcher, 2005; Ramos-Galarza \& Pérez-Salas, 2015; Mayor \& García, 2011; Navarro \& García-Villamisar, 2011).

Las Funciones Ejecutivas (FE) se definen como un conjunto de habilidades cognitivas que permiten establecer objetivos, planificar, iniciar actividades, autorregular y monitorizar las tareas, seleccionar comportamientos y conductas y ejecutar acciones para el logro de los objetivos trazados, y llevar a cabo una conducta eficaz, creativa y aceptada socialmente (Lezak, Howieson, \& Loring, 2004). El curso evolutivo de las FE durante la infancia es decisivo en el funcionamiento cognitivo y en el desarrollo académico, social y afectivo del niño (Sastre-Riba, Merino-Moreno, \& Poch-Olivé, 2007) e involucra el desarrollo de una serie de capacidades cognitivas que le permiten al niño mantener información, manipularla y actuar en función de ésta, autorregular su conducta y adaptarla a los cambios del entorno (García-Molina, Enseñat-Cantallops. Tirapu-Ustárroz, \& Roig-Rovira, 2009; Zelazo, Carter, Reznick, \& Frye, 1997). Por lo que, si hay alteraciones tempranas en el desarrollo ejecutivo, éstas pueden originar diversas consecuencias a corto, mediano y largo plazo (García-Molina et al., 2009).

En los últimos años se ha intentado delimitar los componentes del constructo de las FE identificando, entre ellos, la inhibición, la flexibilidad cognitiva y la planificación y organización (Álvarez, 2006; Corbett, Constantine, Hendren, Rocke, \& Ozonoff, 2009). La inhibición se refiere a la capacidad de impedir la interferencia de información no pertinente ante respuestas en marcha y suprimir informaciones previamente per- 
Pág 101

Un aspecto importante del Trastorno es que, en la mayor parte de los casos, no se presenta solo, sino acompañado de otros síntomas comórbidos (Mateo \& Vilaplana Gramaje, 2007) en asociación con otras entidades psíquicas o del comportamiento, con una frecuencia mayor a la esperada en la población general (Cardo \& Amengual-Gual, 2015). tinentes, pero que no son útiles en la actualidad (Diamond, 2009; Sabagh-Sabbagh, 2008). La inhibición motora se define como la capacidad del individuo de inhibir su respuesta comportamental ante un estímulo (Diamond, 2006), lo que facilita acciones flexibles. Y la inhibición cognitiva se refiere a la supresión de información irrelevante o innecesaria de la memoria de trabajo, pero no de la memoria de reconocimiento (Miyake et al., 2000), lo que hace posible una atención selectiva y sostenida (Diamond, 2006). La flexibilidad cognitiva se define como la capacidad para alternar rápidamente una u otra respuesta, la habilidad de cambiar a estrategias más efectivas entre conjuntos de respuestas, de dividir la atención respondiendo a las demandas cambiantes de una tarea o situación (Anderson, 2002; Mateo \& Vilaplana Gramaje, 2007) y la habilidad para mantener una representación intacta cuando los cambios son irrelevantes (Chevalier \& Blaye, 2008). La planificación y organización se refieren a la capacidad para identificar y organizar una secuencia de eventos con el fin de lograr una meta específica (Rosselli, Jurado, \& Matute, 2008).

Son numerosos los estudios que evidencian que los niños con TDAH presentan un déficit en las FE, como la inhibición del comportamiento (Alloway, 2011; Barkley, 2011; Martel, Nikolas, \& Nigg, 2007; Rubiales, Bakker, \& Urquijo, 2010; Schoemaker et al., 2012), la flexibilidad cognitiva (Martel et al., 2007; Vélez-van-Meerbeke et al., 2013; Ferreiro-Vilasante, Buceta-Cancela, \& Rial-Boubeta, 2013) y la planificación y organización (RamosLoyo, Taracena, Sánchez-Loyo, Matute, \& González-Garrido, 2011; Semrud-Clikeman, Walkowiak, Wilkinson, \& Butcher, 2010). Dicha disfunción ejecutiva se manifiesta a través de un bajo rendimiento en las tareas que evalúan las funciones anteriormente mencionadas, siendo el TDAH un trastorno heterogéneo y complejo, que tiene una gran variabilidad en la manifestación de sus síntomas, los cuales pueden conllevar a dificultades en el ámbito escolar, familiar y social del niño (Chhabildas, Pennington, \& Willcutt, 2001).

Por otro lado, un aspecto importante del Trastorno es que, en la mayor parte de los casos, no se presenta solo, sino acompañado de otros síntomas comórbidos (Mateo \& Vilaplana Gramaje, 2007), en asociación con otras entidades psíquicas o del comportamiento, con una frecuencia mayor a la esperada en la población general (Cardo \& Amengual-Gual, 2015). Precisamente, el TDAH puro es en realidad la presentación más infrecuente del Trastorno, mientras que aproximadamente en $87 \%$ de los casos se asocia con trastornos comportamentales o síntomas comórbidos (Mulas et al. 2006: Ramos-Loyo et al., 2011).

En cuanto a los síntomas comórbidos, estos se pueden diferenciar en síntomas internalizantes, como ansiedad, depresión y obsesiones; y síntomas externalizantes, como problemas de conducta y agresividad (Achenbach, 1985; Navarro \& GarcíaVillamisar, 2011). Entre los síntomas externalizantes, el problema de conducta constituye, junto con las dificultades escolares, la sintomatología con la repercusión más negativa del TDAH (Rodríguez-Molinero et al., 2009). Varios estudios han mostrado que la correlación entre el TDAH y los síntomas externalizantes alcanzan hasta el 20 y 30\%, valores que pueden aumentar hasta 10 puntos al llegar a la adolescencia (Caballo \& Simón, 2001; Wicks-Nelson, Israel, \& Frías, 1997).

Entre los síntomas internalizantes, los más frecuentes en la edad infantil son los síntomas de ansiedad y los depresivos, tales como falta de autoestima, estado de ánimo irritable, falta de energía, somatizaciones y problemas del sueño. Asimismo, debe considerarse que algunos síntomas relacionados con la dificultad para concentrarse son propios tanto del TDAH como del trastorno depresivo (López-Villalobos, SerranoPintado, \& Sánchez-Mateos, 2004). 
Pág 102

Si bien existe una importante cantidad de bibliografía acerca del déficit ejecutivo en niños con TDAH, en la actualidad son escasos los estudios que vinculan un desempeño diferencial del funcionamiento ejecutivo en relación a los síntomas comórbidos que pueden presentarse asociados (Bental \& Tirosh, 2007; Passarotti, Trivedi, \& Patel, 2016; Van De Voorde, Roeyers, Verté, \& Wiersema, 2010; Willcut, Doyle, Nigg, Faraone, \& Pennington, 2005).
Se ha evidenciado que los niños que presentan TDAH asociado con otros síntomas o diagnósticos, presentan una evolución más desfavorable que los niños que tienen TDAH sin comorbilidad, y revisten mayor gravedad clínica, dado que ven afectadas en mayor medida las distintas áreas de su vida familiar, social y académica (LópezVillalobos et al., 2004).

Si bien existe una importante cantidad de bibliografía acerca del déficit ejecutivo en niños con TDAH, en la actualidad son escasos los estudios que vinculan un desempeño diferencial del funcionamiento ejecutivo en relación a los síntomas comórbidos que pueden presentarse asociados (Bental \& Tirosh, 2007; Passarotti, Trivedi, \& Patel, 2016; Van De Voorde, Roeyers, Verté, \& Wiersema, 2010; Willcut, Doyle, Nigg. Faraone, \& Pennington, 2005). En esta línea de investigación, el estudio realizado por Lezama-Espinoza (2015) muestra que al comparar el desempeño ejecutivo de niños con diagnóstico de TDAH con trastornos asociados y niños con diagnóstico de TDAH sin trastornos asociados, no existen diferencias significativas con respecto al funcionamiento ejecutivo, aunque se evidenció un menor rendimiento ejecutivo en el grupo con comorbilidad.

De esta manera, actualmente existen datos contradictorios y poco concluyentes, que no permiten un consenso sobre esta temática, que clarifique si estas condiciones comórbidas inciden, generando un mayor deterioro en las FE o viceversa. Por esta razón, es importante y necesario continuar en la búsqueda de resultados más homogéneos, que aporten a la comprensión del dominio de las FE y su comportamiento en este grupo poblacional

Así, el objetivo del presente estudio es analizar el desempeño en FE en niños con TDAH en función de los síntomas comórbidos asociados, tanto internalizantes como externalizantes.

\section{Método}

\section{Tipo de estudio}

Diseño de tipo ex post facto retrospectivo con un grupo, según la clasificación de Montero y León (2007).

\section{Participantes}

La muestra se conformó por 45 niños escolarizados de la ciudad de Mar del Plata, Argentina, con edades entre 8 y 14 años $(M=10.02 \mathrm{D}=1.92)$, con diagnóstico de TDAH, derivados por médicos neurólogos de centros de salud de la ciudad. Los mismos fueron seleccionados por muestreo no probabilístico por conveniencia. Los criterios de inclusión fueron: presentar diagnóstico de TDAH por médico neurólogo derivante, cumplir con los criterios diagnósticos para el TDAH según el DSM-5, presentar valores por encima del punto de corte esperado en la escala específica de TDAH, y un nivel intelectual (C.I) superior a 70. Se excluyeron niños con antecedentes de enfermedades neurológicas o psiquiátricas, trastornos del aprendizaje y retraso mental.

\section{Instrumentos}

Para la confirmación del diagnóstico médico de TDAH se utilizaron los criterios del DSM-5 (APA, 2013) y la Escala Estandarizada de Swanson, Nolan y Pelham -SNAP IV-, en su versión en español validada en Argentina (Grañana et al., 2006). La misma fue administrada tanto a los padres como a los docentes de los niños. Asimismo, 
para descartar un déficit del nivel intelectual en los niños se administró el Test de Inteligencia de Wechsler para niños (Wechsler, 2010).

Con el propósito de evaluar síntomas comórbidos, se utilizó el listado de síntomas Child Behavior Checklist -CBCL- versión para padres, adaptada y estandarizada en Argentina por Samaniego (2008). Este es un cuestionario tipo Likert con tres opciones de respuesta ( $0=$ No es cierto o nunca; $1=$ Es cierto algunas veces 0 de cierta manera; $2=$ Muy cierto o casi siempre) y está conformado por 113 ítems para evaluar síntomas comórbidos internalizantes (retraimiento, quejas somáticas, síntomas de ansiedad y depresión) y externalizantes (conductas antisociales y agresivas) en niños. Presenta puntos de corte para cada subescala, considerando el puntaje $\mathrm{T}=70$ como el punto de corte aceptado para considerar la presencia de los síntomas evaluados. Es un instrumento que discrimina adecuadamente entre niños referidos a servicios de salud mental y sus pares de población normal, y presenta valores de confiabilidad elevados (.91) junto con altos valores de consistencia interna para las escalas externalizante e internalizante, que va de .85 a .90.

Para evaluar el perfil ejecutivo de los niños se administraron las siguientes pruebas:

- Para evaluar Organización y Planificación se utilizó el test Pirámides de México de la Batería Neuropsicológica Infantil (ENI) (Matute, Rosselli, Ardila, \& Ostrosky-Solís, 2007). El mismo consiste en organizar tres cubos de diferentes colores y tamaño de acuerdo a los diseños solicitados. La tarea consta de 11 ensayos y los movimientos mínimos requeridos para cada ensayo varían de dos a siete. Las variables seleccionadas para establecer las puntuaciones son: diseños correctos, número de movimientos, diseños correctos con mínimos movimientos y tiempo de ejecución en segundos. La prueba presenta un alto coeficiente de confiabilidad entre evaluadores ( $r$.91) y un coeficiente de estabilidad de .24 (Matute et al., 2007).

- Para evaluar la Flexibilidad Cognitiva se aplicó el test Wisconsin Card Sorting Test $( \pm)$ (Heaton, Chelune, Talley, Kay, \& Curtis, 1997). Consta de 64 tarjetas, y el niño tiene que decidir cuál es el principio (color, forma o número) que subyace a la agrupación de las mismas, con la retroalimentación que da el examinador (correcto o incorrecto). Las variables seleccionadas para establecer las puntuaciones son: el número total de tarjetas utilizadas o total de respuestas emitidas; el porcentaje de aciertos, errores, respuestas perseverativas (cuando el niño persiste en responder a una dimensión del estímulo que no es correcta), errores perseverativos, errores no perseverativos, el número de categorías completas y el porcentaje de respuestas de nivel conceptual (series de tres o más respuestas correctas consecutivas) (Heaton et al., 1997; Peña-Casanova, Gramunt, \& Gich, 2005). La prueba presenta una buena fiabilidad (coeficiente de generalizabilidad entre 39 y .72) y evidencias de validez (Heaton et al., 1997).

- Con la finalidad de evaluar el desempeño en Inhibición se aplicó el Test de Stroop de colores y palabras (Golden, 1994) y una Tarea de ejecución-no ejecución (go-no-go) (Soprano, 2003). El Test de Stroop ( \pm ) evalúa la inhibición cognitiva y consta de tres partes: en la primera (Stroop-Palabra), el niño debe leer los nombres de los colores rojo, verde y azul, impresos en negro; en la segunda (Stroop-Color) el niño tiene que denominar los colores impresos en cada fila de ' $x$ ', y en la tercera (Stroop-Palabra Color), que contiene los colores rojo, verde y azul, impresos con un color distinto al que corresponde la palabra escrita, el niño debe nombrar el color de la tinta con la que está impresa la palabra ignorando su significado. La fiabilidad de la prueba con 
Pág 104

El presente estudio se realizó como parte de un proyecto de investigación mayor denominado "Relaciones entre control inhibitorio y regulación emocional en niños con diagnóstico de TDAH" del Grupo de Investigación "Comportamiento Humano, Genética y Ambiente", el cual se encuentra aprobado por la Secretaría de Ciencia y Técnica de la Universidad Nacional de Mar del Plata (UNMDP), Argentina. el empleo del método test-retest es de .89 para Stroop-P, .84 para Stroop-C y .73 para Stroop-PC. Por su parte, la Tarea de ejecución-no ejecución empleada se denomina Golpeteo y se usa para medir la inhibición de funciones motoras, mediante la cual se instruye al niño a responder a un estímulo go (sigue) y a inhibir la respuesta ante el estímulo no-go (para) (Soprano, 2003).

\section{Procedimiento}

El presente estudio se realizó como parte de un proyecto de investigación mayor denominado "Relaciones entre control inhibitorio y regulación emocional en niños con diagnóstico de TDAH" del Grupo de Investigación "Comportamiento Humano, Genética y Ambiente", el cual se encuentra aprobado por la Secretaría de Ciencia y Técnica de la Universidad Nacional de Mar del Plata (UNMDP), Argentina. En el marco de convenios firmados con dos Instituciones de Salud de la ciudad de Mar del Plata, y a partir de los datos proporcionados por los médicos derivadores, se establecieron los contactos con los niños con TDAH y sus padres, a quienes se los citó en las instalaciones del Instituto de Psicología Básica, Aplicada y Tecnología (IPSIBAT) de la Facultad de Psicología de la UNMDP para realizar la evaluación individual. Se les solicitó a los padres que, durante el periodo de evaluación y durante las 24 horas previas a la misma, los niños que se encontraban en tratamiento farmacológico no recibiesen medicación. La participación fue voluntaria y sujeta al asentimiento de los niños y al consentimiento informado de los padres. Durante el desarrollo del trabajo se respetaron los principios éticos de la investigación con seres humanos, procurándose las condiciones necesarias para proteger la confidencialidad y actuar en beneficio de los participantes.

\section{Análisis estadístico}

Para realizar los análisis estadísticos se utilizó el paquete estadístico SPSS, versión 19. Con el objeto de describir el funcionamiento ejecutivo en niños con TDAH de acuerdo a la ausencia o presencia de síntomas comórbidos internalizantes y externalizantes, los datos obtenidos fueron sometidos a un análisis estadístico descriptivo de media (M) y desvío estándar (DE). Asimismo, con el propósito de establecer si las diferencias observadas eran estadísticamente significativas, se sometieron los datos a la prueba ANOVA de un factor con la prueba post hoc de DMS (diferencia menos significativa), para realizar las comparaciones entre las medias de los grupos, en función de la ausencia o presencia de síntomas comórbidos internalizantes y externalizantes. Adicionalmente, se calculó, para cada una de las variables, el tamaño del efecto de la diferencia, utilizando la clasificación por rangos de Cohen, el cual indica que el tamaño del efecto puede ser grande (mayor a .8), mediano (cercano a .5) o menor (menor a .2) (Quezada, 2007). Por último, se realizó un análisis discriminante con el fin de identificar las características o variables que permiten diferenciar y clasificar a los grupos.

\section{Resultados}

En la tabla 1 se presentan los estadísticos descriptivos para la variable flexibilidad cognitiva discriminados en función de la pertenencia al grupo y los resultados de la ANOVA con el tamaño del efecto correspondiente.

Puede observarse que en la prueba de flexibilidad cognitiva (WCST) existen diferencias estadísticamente significativas entre el grupo de niños sin síntomas comórbidos (TDAH-P) y el grupo de niños con síntomas internalizantes (TDAH-I) con respecto al porcentaje de Errores no Perseverativos, con un tamaño del efecto mediano. 
Pág 105

Tabla 1. Estadísticos descriptivos del Wisconsin Card Sorting Test (WCST) en función de los grupos de niños sin síntomas comórbidos (TDAH-P), niños con síntomas internalizantes (TDAH-I) y niños con síntomas externalizantes (TDAH-E), y resultados de la ANOVA y tamaño del efecto Cohen's d.

\begin{tabular}{|c|c|c|c|c|c|c|c|c|c|c|c|c|c|}
\hline \multicolumn{2}{|c|}{ Flexibilidad Cognitiva } & \multicolumn{6}{|c|}{ Descriptivos } & \multicolumn{6}{|c|}{ ANOVA } \\
\hline \multirow[t]{2}{*}{ Test } & \multirow[t]{2}{*}{ Variables } & \multicolumn{2}{|c|}{ TDAH-P } & \multicolumn{2}{|c|}{ TDAH-I } & \multicolumn{2}{|c|}{ TDAH-E } & \multicolumn{2}{|c|}{$\begin{array}{l}\text { TDAH-P } \\
\text { TDAH-I }\end{array}$} & \multicolumn{2}{|c|}{$\begin{array}{l}\text { TDAH-P } \\
\text { TDAH-E }\end{array}$} & \multicolumn{2}{|c|}{$\begin{array}{l}\text { TDAH-I } \\
\text { TDAH-E }\end{array}$} \\
\hline & & $M$ & $D E$ & $M$ & $D E$ & $M$ & $D E$ & Sig. & $d$ & Sig. & $d$ & Sig. & d \\
\hline & Respuestas & 55.52 & 10.59 & 55.30 & 11.30 & 54.10 & 9.18 & .96 & .02 & .72 & .14 & .80 & .11 \\
\hline & $\%$ Errores & 31.90 & 15.17 & 27.62 & 7.91 & 30.27 & 14.92 & .41 & .35 & .76 & .10 & .67 & .22 \\
\hline & Categorías & 2.24 & .83 & 2.30 & .67 & 2.30 & .82 & .84 & .07 & .84 & .07 & 1.00 & .00 \\
\hline & \% Perseveraciones & 18.45 & 16.36 & 17.47 & 8.98 & 20.51 & 15.36 & .86 & .07 & .71 & .12 & .65 & .24 \\
\hline
\end{tabular}

* La correlación es significante al nivel .05 (bilateral).

En la tabla 2 pueden observarse los estadísticos descriptivos para la variable organización y planificación discriminados en función de la pertenencia al grupo y los resultados de la ANOVA con el tamaño del efecto correspondiente.

Como puede observarse en la tabla 2, en la prueba de organización y planificación (TPM) existen diferencias estadísticamente significativas tanto entre el grupo de niños sin síntomas comórbidos (TDAH-P) y el grupo de niños con síntomas internalizantes (TDAH-I), como entre el grupo de niños con síntomas internalizantes (TDAH-I) y el grupo de niños con síntomas externalizantes (TDAH-E), con respecto a Diseños correctos, con un tamaño del efecto grande.

Tabla 2. Estadísticos descriptivos del Test Pirámides de México (TPM) en función de los grupos de niños sin síntomas comórbidos (TDAH-P), niños con síntomas internalizantes (TDAH-I) niños con síntomas externalizantes (TDAH-E), y resultados de la ANOVA y tamaño del efecto Cohen's d.

\begin{tabular}{|c|c|c|c|c|c|c|c|c|c|c|c|c|c|}
\hline \multicolumn{2}{|c|}{ Organización y Planificación } & \multicolumn{6}{|c|}{ Descriptivos } & \multicolumn{6}{|c|}{ ANOVA } \\
\hline \multirow{2}{*}{ Test } & \multirow{2}{*}{ Variables } & \multicolumn{2}{|c|}{ TDAH-P } & \multicolumn{2}{|c|}{ TDAH-I } & \multicolumn{2}{|c|}{ TDAH-E } & \multicolumn{2}{|c|}{$\begin{array}{c}\text { TDAH-P } \\
\text { TDAH- }\end{array}$} & \multicolumn{2}{|c|}{$\begin{array}{l}\text { TDAH-P } \\
\text { TDAH-E }\end{array}$} & \multicolumn{2}{|c|}{$\begin{array}{l}\text { TDAH-I } \\
\text { TDAH-E }\end{array}$} \\
\hline & & $M$ & $D E$ & $M$ & $D E$ & $M$ & $D E$ & Sig. & $d$ & Sig. & $d$ & Sig. & $d$ \\
\hline & Diseños correctos & 10.40 & 1.22 & 9.10 & 1.44 & 10.60 & .69 & $.01^{* *}$ & .97 & .66 & .20 & $.01^{* *}$ & 1.32 \\
\hline & $\mathrm{N}^{0}$ de movimientos & 66.08 & 12.68 & 68.00 & 15.75 & 63.30 & 12.25 & .70 & .13 & .58 & .22 & .43 & .33 \\
\hline & Dis.Corr/Min.Mov & 9.36 & 12.28 & 6.10 & 2.60 & 7.60 & 2.01 & .36 & .36 & .62 & .20 & .72 & .64 \\
\hline$\underset{1}{2}$ & Tiempo en seg. & 141.02 & 82.02 & 125.43 & 46.35 & 119.37 & 37.05 & .54 & .23 & .40 & .34 & .84 & .14 \\
\hline
\end{tabular}

** La correlación es significativa al nivel .01 (bilateral) 
Y respecto a los resultados de la variable inhibición, en la tabla 3 pueden observarse los estadísticos descriptivos discriminados en función de la pertenencia al grupo y los resultados de la ANOVA con el tamaño del efecto correspondiente.

En la tabla 3 se observa que en la prueba de inhibición cognitiva (TS) existen diferencias estadísticamente significativas tanto entre el grupo de niños sin síntomas comórbidos (TDAH-P) y el grupo de niños con síntomas internalizantes (TDAH-I) como entre el grupo de niños con síntomas internalizantes (TDAH-I) y el grupo de niños con síntomas externalizantes (TDAH-E) con respecto a interferencia, con un tamaño del efecto grande. Por otro lado, se observa que en la prueba de inhibición motora (TENE) existen diferencias estadísticamente significativas entre el grupo de niños sin síntomas comórbidos (TDAH-P) y el grupo de niños con síntomas externalizantes (TDAH-E), con un tamaño del efecto mediano.

Tabla 3. Estadísticos descriptivos del Test de Stroop (TS) y la Tarea de ejecución-no ejecución (TENE) en función de los grupos de niños sin síntomas comórbidos (TDAH-P), niños con síntomas internalizantes (TDAH-I) y

niños con síntomas externalizantes (TDAH-E), y resultados de la ANOVA y tamaño del efecto Cohen's d.

\begin{tabular}{|c|c|c|c|c|c|c|c|c|c|c|c|c|c|}
\hline \multirow{3}{*}{ Test } & \multirow{3}{*}{$\begin{array}{c}\text { Inhibición } \\
\text { Variables }\end{array}$} & \multicolumn{6}{|c|}{ Descriptivos } & \multicolumn{6}{|c|}{ ANOVA } \\
\hline & & \multicolumn{2}{|c|}{ TDAH-P } & \multicolumn{2}{|c|}{ TDAH-I } & \multicolumn{2}{|c|}{ TDAH-E } & \multicolumn{2}{|c|}{$\begin{array}{l}\text { TDAH-P } \\
\text { TDAH-I }\end{array}$} & \multicolumn{2}{|c|}{$\begin{array}{l}\text { TDAH-P } \\
\text { TDAH-E }\end{array}$} & \multicolumn{2}{|c|}{$\begin{array}{l}\text { TDAH-I } \\
\text { TDAH-E }\end{array}$} \\
\hline & & M & $D E$ & M & $D E$ & M & $D E$ & Sig. & $d$ & Sig. & $d$ & Sig. & $D$ \\
\hline \multirow{4}{*}{ TS } & Palabra & 43.80 & 6.42 & 44.40 & 5.23 & 44.20 & 9.05 & .82 & .10 & .88 & .05 & .95 & .02 \\
\hline & Color & 40.68 & 8.15 & 43.00 & 4.83 & 43.20 & 9.80 & .44 & .34 & .40 & .27 & .96 & .02 \\
\hline & Palabra-Color & 40.64 & 9.59 & 40.70 & 3.88 & 45.50 & 6.72 & .98 & .00 & .12 & .58 & .19 & .87 \\
\hline & Interferencia & 49.36 & 7.78 & 44.30 & 4.32 & 50.50 & 5.52 & $.04^{*}$ & .80 & .65 & .16 & $.04^{*}$ & 1.25 \\
\hline TENE & Golpeteo & 9.52 & .71 & 8.50 & 2.06 & 8.20 & 2.74 & .11 & .66 & $.04^{*}$ & .65 & .69 & .12 \\
\hline
\end{tabular}

* La correlación es significante al nivel .05 (bilateral)

** La correlación es significativa al nivel .01 (bilateral)

Finalmente, con el propósito de determinar si el funcionamiento de las variables analizadas permite diferenciar y clasificar a los grupos, en la tabla 4 se presentan los resultados de un análisis discriminante y en la tabla 5 se presentan los resultados de la Matriz de estructura del análisis discriminante con el peso relativo de cada variable.

Tabla 4. Clasificación del análisis discriminante de los niños con TDAH en función de los grupos

\begin{tabular}{|c|c|c|c|c|c|c|c|c|c|}
\hline & & \multicolumn{8}{|c|}{ Grupo de pertenencia pronosticado } \\
\hline & & \multicolumn{2}{|c|}{ TDAH-P } & \multicolumn{2}{|c|}{ TDAH-I } & \multicolumn{2}{|c|}{ TDAH-E } & \multicolumn{2}{|c|}{ Total } \\
\hline & & Recuento & $\%$ & Recuento & $\%$ & Recuento & $\%$ & Recuento & $\%$ \\
\hline \multirow{3}{*}{ GruposOriginal } & TDAH-P & 25 & 100 & 0 & .0 & 0 & .0 & 25 & 100 \\
\hline & TDAH-I & 2 & 20 & 7 & 70 & 1 & 10 & 10 & 100 \\
\hline & TDAH-E & 3 & 30 & 1 & 10 & 6 & 60 & 10 & 100 \\
\hline
\end{tabular}

a. Clasificados correctamente el $84,4 \%$ de los casos agrupados originales 
A partir del análisis discriminante realizado con las variables cognitivas analizadas anteriormente, se observa que las mismas presentan una sensibilidad del $84,4 \%$, es decir, que permiten discriminar correctamente la pertenencia al grupo con síntomas comórbidos internalizante, al grupo con síntomas comórbidos externalizante y al grupo sin síntomas comórbidos en el 84,4\%.

Tabla 5. Matriz de estructura del análisis discriminante

\begin{tabular}{|c|c|c|c|}
\hline \multirow{2}{*}{ Tests } & \multirow{2}{*}{ Variables } & \multicolumn{2}{|c|}{ Función } \\
\hline & & 1 & 2 \\
\hline TPM & Diseños correctos & $.35^{*}$ & .18 \\
\hline TS & Interferencia & $.24^{\circ}$ & .16 \\
\hline WCST & $\%$ Aciertos & $-.11^{\circ}$ & .11 \\
\hline WCST & \% Errores & $.08^{*}$ & -.05 \\
\hline TENE & Golpeteo & .14 & $-.47^{*}$ \\
\hline TS & Palabra-Color & .03 & $.39^{*}$ \\
\hline WCST & \% Errores no Pers. & .24 & $-.27^{*}$ \\
\hline TPM & Tiempo en seg. & .05 & $-.19^{*}$ \\
\hline TS & Color & -.07 & $.18^{*}$ \\
\hline TPM & $\mathrm{N}^{\circ}$ de movimientos & -.05 & $-.14^{*}$ \\
\hline TPM & Dis.Corr/Min.Mov. & .09 & $-.09^{*}$ \\
\hline WCST & \% Perseveraciones & .02 & $.09^{*}$ \\
\hline WCST & Respuestas & -.00 & $-.08^{*}$ \\
\hline WCST & \% Errores Pers. & .02 & $.06^{*}$ \\
\hline WCST & Categorías & -.01 & $.04^{*}$ \\
\hline TS & Palabra & -.02 & $.03^{*}$ \\
\hline
\end{tabular}

En la tabla 5 puede observarse el peso relativo de cada variable a la función discriminante, donde se presentan las variables ordenadas por el tamaño de la correlación con la función. Se observan mayores tamaños en los valores referidos a la Tarea de ejecución-no ejecución (TENE), la variable Palabra-Color del Test de Stroop (TS) y la variable Diseños Correctos del Test Pirámides de México (TPM), lo cual indica que estos son los puntajes que mejor permiten discriminar la pertenencia al grupo con síntomas comórbidos internalizantes, al grupo con síntomas comórbidos externalizantes y al grupo sin síntomas comórbidos.

\section{Discusión y Conclusiones}

El objetivo del presente estudio fue analizar el desempeño en FE en niños con TDAH en función de los síntomas comórbidos asociados, tanto internalizantes como externalizantes.

Los resultados evidencian que existen diferencias entre el grupo de niños sin síntomas comórbidos (TDAH-P) y el grupo de niños con síntomas internalizantes (TDAH-I) con respecto al desempeño en flexibilidad cognitiva (Porcentaje de Errores no Perseverativos), en organización y planificación (Diseños correctos) y en inhibición cognitiva (Interferencia); y en inhibición motora. Por lo que se observa un desempeño ejecutivo diferencial entre ambos grupos, así: el grupo de niños con TDAH-I presenta un mejor desempeño en flexibilidad e inhibición cognitiva y un peor desempeño en 
Los resultados señalaron que las variables ejecutivas analizadas permiten discriminar de modo satisfactorio la pertenencia a cada uno de los grupos, indicando una posible selección de variables que permitirían colaborar con el proceso de evaluación diagnóstica del trastorno. organización y planificación y en inhibición motora, en comparación con el grupo de niños con TDAH-P. Asimismo, se encontraron diferencias entre el grupo de niños con TDAH-P y el grupo de niños con síntomas externalizantes (TDAH-E) en inhibición motora, teniendo un desempeño inferior el grupo de niños con TDAH-E.

Por otro lado, los resultados muestran que existen diferencias entre el grupo de niños con TDAH-l y el grupo de niños con TDAH-E con respecto al desempeño en organización y planificación (Diseños correctos) y en inhibición cognitiva (Interferencia), observándose que el grupo de niños con TDAH-E presenta un mejor desempeño en organización y planificación y un peor desempeño en inhibición cognitiva, en comparación con el grupo de niños con TDAH-I.

Asimismo, los resultados señalaron que las variables ejecutivas analizadas permiten discriminar de modo satisfactorio la pertenencia a cada uno de los grupos, indicando una posible selección de variables que permitirían colaborar con el proceso de evaluación diagnóstica del trastorno.

Estos resultados coinciden con un estudio que indica que los niños con diagnóstico de TDAH con comorbilidad presenta desempeños inferiores en FE en comparación a los niños con TDAH sin comorbilidad (Passarotti et al., 2016) y se contraponen a los estudios que no evidencian diferencias significativas con respecto al funcionamiento ejecutivo en niños con TDAH con y sin presencia de síntomas comórbidos, aunque evidencian, en consonancia con el presente trabajo, un menor rendimiento ejecutivo en el grupo con comorbilidad (Willcut et al., 2005; Lezama-Espinoza, 2015).

El Modelo Híbrido de las Funciones Ejecutivas (Barkley, 1997) y su posterior reformulación en el Modelo de Autorregulación de Barkley (1998) podría explicar los resultados encontrados, en relación a que los niños con TDAH tanto con sintomatología externalizante como internalizante presenten dificultades en el control inhibitorio, al ser éste el mecanismo cognitivo básico que afecta de manera jerárquica otras áreas que requieren un control inhibitorio adecuado para ejecutarse efectivamente, como la regulación comportamental y cognitiva (Antshel, Hier, \& Barkley, 2014; HenríquezHenríquez, Zamorano-Mendieta, Rothhammer-Engel, \& Aboitiz, 2010). Así, se observan dificultades para desarrollar conductas orientadas hacia un objetivo, reducida capacidad de persistencia, de control de la conducta y de la activación emocional, insensibilidad al feedback de proceso y resultado, desorganización en las tareas y afectación por aspectos irrelevantes de las mismas (Campeño-Martínez, 2013; Servera-Barceló, 2005).

El estudio de subgrupos de niños con diagnóstico de TDAH con síntomas comórbidos, con otros trastornos externalizantes e internalizantes, es una necesidad clave para entender en profundidad la naturaleza de los problemas que padecen estos niños (Spencer, 2005). La presencia de síntomas comórbidos en los niños con TDAH suele ser una regla común, por lo cual implica la necesidad de ajustes en los procesos diagnósticos a partir de un completo diagnóstico diferencial y en las intervenciones terapéuticas posteriores (Castellanos \& Acosta, 2004). Delimitar y perfilar el desempeño ejecutivo que puede subyacer al TDAH y sus diversas asociaciones comórbidas, posibilitaría un mejor conocimiento de la entidad, lo cual resultaría de importancia para ser considerado al momento de iniciar el proceso diagnóstico, colaborando en un adecuado diagnóstico diferencial, y en la planificación de un tratamiento integral adaptado a las necesidades y problemáticas de cada niño (Cardo \& Servera, 2008). 
Una línea de trabajo que se desprende del presente estudio es analizar la relación entre el desempeño ejecutivo y las comorbilidades, en función de los subtipos diagnósticos del trastorno. Asimismo, otra línea de interés podría estar vinculada al desarrollo de instrumentos de evaluación que aporten, por un lado, más precisión en las funciones evaluadas, permitiendo mayor fiabilidad para relacionar el desempeño con posibles alteraciones funcionales, y, por otro lado, validez ecológica referida a que el desempeño en la prueba posibilite inferir la capacidad funcional del niño en sus actividades cotidianas.

Es importante mencionar que una de las limitaciones de este trabajo está relacionada con el tamaño de la muestra, debido a los inconvenientes que se presentan habitualmente para evaluar pacientes diagnosticados con alguna patología y que cumplan con todos los criterios de inclusión que se requieren en un diseño de investigación. Para el presente estudio fueron controladas las características ya indicadas respecto al proceso diagnóstico, sin embargo, sería recomendable para futuros estudios ampliar la muestra a un número mayor, que permita generalizar los datos de acuerdo a estadísticas nacionales (Argentina) de prevalencia del trastorno.

\section{Referencias}

Achenbach, T. M. (1985). Assesment and Taxonomy of Child Adolescent Psychopathology. London: Sage.

Alloway,T.P.(2011).Acomparison of working memory profiles in children with ADHDand DCD. Child Neuropsychology, 17(5), 483-494. doi: 10.1080/09297049.2011.553590.

Álvarez, J. B. (2006). Diagnóstico del trastorno de déficit de atención con/sin hiperactividad. Una visión desde la evidencia científica. Revista Pediatría de Atención Primaria, 8(4), 25-37. Recuperado de http://www.pap.es/files/1116-591-pdf/616. pdfdoi: 10.1080/09297049.2011.553590.

Anderson, P. (2002). Assessment and development of executive function (EF) during childhood. Child Neuropsychology, 8(2), 71-82. doi: 10.1076/chin.8.2.71.8724.

Antshel, K. M., Hier, B. O., \& Barkley, R. A. (2014). Executive functioning theory and adhd. En S. Goldstein, \& J. A. Naglieri (Eds.), Handbook of Executive Functioning (107-120). New York: Springer. http://link.springer.com/book/10.1007\% 2F978-1-4614-8106-5

Asociación Americana de Psiquiatría (APA). (2013). Diagnostic and Statistical Manual of Mental Disorders (5 ed.). Washinton DC: Arlington, VA, American Psychiatric Publishing. http://dsm.psychiatryonline.org/doi/book/10.1176/appi. books. 9780890425596

Bakker, L., \& Rubiales, J. (2010). Interacción de factores genéticos y ambientales en el trastorno por déficit de atención con hiperactividad. Estudio de caso de gemelos. Revista Chilena de Neuropsicología, 5(3), 226-236. Recuperado de http:// fundacioncadah.org/j289eghfd7511986 uploads/20120607 n0t8KUPZUcP2uigNIKE9 $0 . p d f$

Barkley, R. A. (2011). Is executive functioning deficient in ADHD? It depends on your definitions and your measures. The ADHD Report, 19(4), 1-10. doi: 10.1521/ adhd.2011.19.4.1

Barkley, R. A. (1997). Behavioral inhibition, sustained attention, and executive functions: constructing a unifying theory of ADHD. Psychological Bulletin, 121, 65-94. doi: 10.1037/0033-2909.121.1.65

Barkley, R. A. (1998). A theory of ADHD: Inhibition, executive functions, selfcontrol, and time. En R. A. Barkley (Ed.), Attention Deficit Hyperactivity Disorders: A Handbook for Diagnosis and Treatment (225-262). New York: Guilford. http://revistas. ces.edu.co/index.php/psicologia/article/view/3640 
Bental, B., \& Tirosh, E. (2007). The relationship between attention, executive functions and reading domain abilities in attention deficit hyperactivity disorder and reading disorder: a comparative study. Journal of the American Academy of Child \& Adolescent Psychiatry, 48(5), 455-463. doi: 10.1111/j.1469-7610.2006.01710.x

Caballo,V., \& Simón, M. (2001). Manual de Psicología:Clínica Infantily del Adolescente. Madrid: Pirámide. http://www.casadellibro.com/libro-manual-de-psicologia-clinica-infantil-y-del-adolescente-trastorn-os-especificos/9788436816419/821298

Campeño Martínez, Y. (2013). Intervención psicopedagógica en niños afectados por el síndrome TDAH. Estudio experimental. (Tesis Doctoral). Universidad Complutense de Madrid, España. http://eprints.ucm.es/24523/1/T35110.pdf

Cardo, E., \& Amengual-Gual, M. (2015). ¿Se asocia el trastorno por déficit de atención/hiperactividad con otras patologías prevalentes de la infancia?. Revista de Neurología, 60(1), 109-113. Recuperado de http://www.neurologia.com/pdf/ Web/60S01/bnS01S109.pdf

Cardo, E. \& Servera, M. (2008). Trastorno por déficit de atención/hiperactividad: estado de la cuestión y futuras líneas de investigación. Revista de Neurología, 46(6), 365-372. Recuperado de http://faros.hsjdbcn.org/adjuntos/130.2-tdha.pdf

Castellanos, F. X., \& Acosta, M. T. (2004). Síndrome de Tourette: análisis de la comorbilidad y sus tratamientos específicos. Revista de Neurología, 38(1), 124-130. Recuperado de https://dialnet.unirioja.es/servlet/articulo?codigo $=4659928$

Catelan-Mainardes, S. C. (2010). Transtorno de déficit de atenção e hiperatividade na infância e adolescência pela perspectiva da neurobiologia. Revista Saúde e Pesquisa, 3(3), 385-391. Recuperado de http://periodicos.unicesumar.edu.br/index. php/saudpesq/article/view/1473

Chevalier, N., \& Blaye, A. (2008). Cognitive flexibility in preschoolers: The role of representation activation and maintenance. Developmental Science, 11, 339-353. doi: 10.1111/j.1467-7687.2008.00679.x

Chhabildas, N., Pennington, B. F., \& Willcutt, E. G. (2001). A Comparison of the neuropsychological profiles of the DSM-IV subtypes of ADHD. Journal of Abnormal Child Psychology, 29(6), 529-540. Recuperado de http://link.springer.com/ article/10.1023/A:1012281226028

Corbett, B. A., Constantine, L. J., Hendren, R., Rocke, D., \& Ozonoff, S. (2009). Examining executive functioning in children with autism spectrum disorder, attention deficit hyperactivity disorder and typical development. Psychiatry Research, 166, 210-222. doi: 10.1016/j.psychres.2008.02.005

De la Peña, F., Palacio, J. D., \& Barragán, E. (2010). Declaración de Cartagena para el Trastorno por Déficit de Atención con Hiperactividad (TDAH): rompiendo el estigma. Revista Ciencias de la Salud, 8(1), 93-98. Recuperado de http://www. scielo.org.co/pdf/recis/v8n1/v8n1a9

Diamond, A. (2006). The early development of executive functions. En E. Bialystok, \& F. Craik, (Eds.), Lifespan Cognition: Mechanisms of Change (70-95). New York: Oxford University Press. http://www.devcogneuro.com/Publications/Diamond\%20 2014\%20Intro\%20Spring\%202014\%20Final.pdf

Diamond, A. (2009). All or none hypothesis: A global-default mode that characterizes the brain and mind. Developmental Psychology, 45(1), 130-138. doi: 10.1037/a0014025

Ferreiro-Vilasante, M., Buceta-Cancela, M. J., \& Rial-Boubeta, A. (2013). Comparación de la flexibilidad cognitiva en el TDAH y la dislexia. Infancia y Aprendizaje, 36(1), 105-117. doi: 10.1174/021037013804826500

Fischer, M., Barkley, R. A., Smallish, L., \& Fletcher, K. (2005). Executive functioning in hyperactive children as young adults: attention, inhibition, response perseveration, and the impact of comorbidity. Developmental Neuropsychology, 27(1), 107133. doi: $10.1207 /$ s15326942dn2701 5 
Gaitán-Chipatecua, A., \& Rey-Anacona, C. (2013). Diferencias en funciones ejecutivas en escolares normales, con trastorno por déficit de atención e hiperactividad, trastorno del cálculo y condición comórbida. Avances en Psicología Latinoamericana, 31(1), 71-85. Recuperado de http://www.scielo.org.co/pdf/apl/v31n1/ v31n1a06.pdf

García-Molina, A. A., Enseñat-Cantallops, J., Tirapu-Ustárroz, T., \& Roig-Rovira. (2009). Maduración de la corteza prefrontal y desarrollo de las funciones ejecutivas durante los primeros cinco años de vida. Revista de Neurología, 48(8), 435-440. Recuperado de http://www.neurologia.com/pdf/Web/4808/bb080435.pdf

Golden, C. J. (1994). STROOP: Test de Colores y Palabras: Manual. España: TEA. http:// web.teaediciones.com/stroop-test-de-colores-y-palabras.aspx

Grañana, N., Richaudeau, A., Robles Gorriti, C., Scotti, M., Fejerman, N., \& Allegri, R. (2006). Detección de síntomas para trastorno por déficit de atención e hiperactividad: escala SNAP IV, validación en Argentina. Revista Neurológica Argentina, 28(1), 20. http://www.aidep.org/03 ridep/R38/Art2.pdf

Heaton, R., Chelune, G., Talley, J., Kay, G., \& Curtis, G. (1997). Test de Clasificación de Tarjetas de Wisconsin. Madrid: TEA. http://web.teaediciones.com/wcst-test-declasificacion-de-tarjetas-de-wisconsin.aspx

Henríquez-Henríquez, M., Zamorano-Mendieta, F., Rothhammer-Engel, F., \& Aboitiz, F. (2010). Modelos neurocognitivos para el trastorno por déficit de atención/hiperactividad y sus implicaciones en el reconocimiento de endofenotipos. Revista de Neurología, 50(2), 109-116. Recuperado de http://www.neurologia.com/pdf/ Web/5002/bd020109.pdf

Labos, E., Slachevsky, A., Fuentes, P., \& Manes, F. (2008). Tratado de Neuropsicología clínica. Buenos Aires: Akadia. http://www.editorialakadia.com/index. php? route $=$ product $/$ product $\&$ product id $=289$

Lezak, M. D., Howieson, D. V., \& Loring, D. W. (2004). Neuropsychological Assessment. (4ed). New York: Oxford University Press. https://global.oup.com/academic/ product/neuropsychological-assessment-9780195395525?cc=us\&lang=en\&

Lezama-Espinoza, E. A. (2015). Relación de las funciones ejecutivas con trastornos externalizantes y/o internalizantes en niños de 5 a 15 años con TDAH que asisten a programas de atención a la población infantil de la ciudad de Manizales (Tesis Doctoral). Universidad Autónoma de Manizales, Colombia.

López-Villalobos, J., Serrano-Pintado, I., \& Sánchez-Mateos, J. (2004). Trastorno por déficit de atención con hiperactividad: comorbilidad con trastornos depresivos y de ansiedad. Psicothema, 16(3), 402-407. Recuperado de http://www.unioviedo. net/reunido/index.php/PST/article/view/8214

Martel, M., Nikolas, M., \& Nigg, J. T. (2007). Executive function in adolescents with ADHD. Journal of the American Academy of Child and Adolescent Psychiatry, 46, 1437-1444. doi: 10.1097/chi.0b013e31814cf953

Mateo, F. M., \& Vilaplana Gramaje, A. (2007). Estrategias de identificación del alumno inatento e impulsivo desde el contexto escolar. Quaderns Digitals, 5, 13-28. Recuperado de http://www.quadernsdigitals.net/datos/hemeroteca/r 72/ nr 769/a 10377/10377.pdf

Matute, E., Rosselli, M., Ardila, A., \& Ostrosky-Solís, F. (2007). Evaluación Neuropsicológica Infantil. México: Manual Moderno. http://web.uaemex.mx/sa/rm/docs/ catalogos/CATALOGOS\%20MANUAL\%20MODERNO/CATALOG0\%20MANUAL\%20MODERN0\%20Instrumentosdeevaluacion.pdf

Mayor, J. G. \& García, R. S. (2011). Trastorno por Déficit de Atención/Hiperactividad (TDAH) Revisión ¿Hacia dónde vamos ahora?. Revista Chilena de Psiquiatría y Neurología de la Infancia y la Adolescencia, 22(2), 144-154. Recuperado de http:// www.sopnia.com/boletines/Revista\%20SOPNIA\%202011-2.pdf\#page=34 
Miyake, A., Friedman, N. P., Emerson, M. J., Witzki, A. H., Howerter, A., \& Wager, T. D. (2000). The Unity and Diversity of Executive Functions and Their Contributions to Complex "Frontal Lobe" Tasks: A Latent Variable Analysis. Cognitive Psychology, 41, 49-100. doi:10.1006/cogp.1999.0734

Montero, I., \& León, O. (2007). A guide for naming research studies in Psychology. International Journal of Clinical and Health Psychology, 7(3), 847-862. Recuperado de http://www.aepc.es/ijchp/GNEIP07 es.pdf

Mulas, F., Abad Mas, L., Etchepareborda, M. C., Díaz-Lucero, A., Hernández, S., RuizAndrés, R., ... Pascuale, M. J. (2006). Trastornos neuropsicológicos de los adolescentes afectos de trastorno por déficit de atención con hiperactividad. Revista de Neurología, 43(1), 71-81. Recuperado de https://dialnet.unirioja.es/servlet/ articulo?codigo $=4696566$

Navarro, M. I., \& García-Villamisar, D. A. (2011). Funcionamiento ejecutivo en el trastorno de déficit de atención con hiperactividad: una perspectiva ecológica de los perfiles diferenciales entre los tipos combinado e inatento. Revista de Psicopatología y Psicología Clínica, 16(2), 113-124. doi: 10.5944/rppc.vol.16. num.2.2011.10355

Passarotti, A. M., Trivedi, N., \& Patel, M. (2016). Executive function in adolescent bipolar disorder with and without ADHD comorbidity. Bipolar Disorder, 1(1), 101. doi:10.4172/jbd.1000101

Peña-Casanova, J., Gramunt, N., \& Gich, J. (2005). Test Neuropsicológicos. Barcelona: Masson. http://web.uaemex.mx/sa/rm/docs/catalogos/CATALOGOS\%20MANUAL\%20MODERNO/CATALOGO\%20MANUAL\%20MODERN0\%20Instrumentosdeevaluacion.pdf

Quezada, C. (2007). Potencia estadística, sensibilidad y tamaño de efecto: ¿un nuevo canon para la investigación?. Onomázein, 16, 159-170. Recuperado de http:// www.onomazein.net/Articulos/16/4 Quezada.pdf

Ramos Galarza, C. A., \& Pérez Salas, C. P. (2015). Relación entre el modelo híbrido de las funciones ejecutivas y el Trastorno por déficit de atención con hiperactividad. Psicología desde el Caribe, 32(2), 299-314. Recuperado de http://www. scielo.org.co/pdf/psdc/v32n2/v32n2a08.pdf

Ramos-Loyo, J., Taracena, A, M., Sánchez-Loyo, L. M., Matute, E., \& González-Garrido, A. A. (2011). Relación entre el funcionamiento ejecutivo en pruebas neuropsicológicas y en el contexto social en niños con TDAH. Revista Neuropsicología, Neuropsiquiatría y Neurociencias, 11(1), 1-16. Recuperado de https://dialnet. unirioja.es/servlet/articulo?codigo $=3640848$

Rodillo, B. E. (2015). Trastorno por déficit de atención e hiperactividad (TDAH) en adolescentes. Revista Médica Clínica Las Condes, 26(1), 52-59. doi: 10.1016/j.rmclc.2015.02.005

Rodríguez-Molinero, L., López-Villalobos, J. M., Garrido-Redondo, M., Sacristán-Martín, A. M., Martínez-Rivera, M. T., \& Ruiz-Sanz, F. (2009). Estudio psicométricoclínico de prevalencia y comorbilidad del Trastorno por déficit de atención con hiperactividad en Castilla y León (España). Revista Pediatría de Atención Primaria, 11(42), 251-270. Recuperado de http://scielo.isciii.es/pdf/pap/v11n42/06 originales.pdf

Rosselli, M., Jurado, M. B., \& Matute, E. (2008). Las funciones ejecutivas a través de la vida. Revista de Neuropsicología, Neuropsiquiatría y Neurociencias, 8(1), 23-46. Recuperado de https://dialnet.unirioja.es/servlet/articulo?codigo=3987451

Rubiales, J., Bakker, L., \& Urquijo, S. (2010). Inhibición cognitiva y motora en niños con Trastorno por déficit de atención con hiperactividad. Revista Acta Psiquiátrica y Psicológica de América Latina, 56(2), 75-82. Recuperado de http://www.acta. org.ar/04-WebForms/frmResumen.aspx?IdArticulo=164\&Abonado= 
Sabagh-Sabbagh, S. (2008). Solution of written arithmetic problems and inhibitory cognitive control. Universitas Psychologica, 7(1), 215-228. Recuperado de http:// www.scielo.org.co/pdf/rups/v7n1/v7n1a16.pdf

Samaniego, V. C. (2008). El child behavior check list: su estandarización en población urbana Argentina. Revista de Psicología UCA, 4(8), 113-130. Recuperado deResearchgate

Sastre-Riba, S., Merino-Moreno, N., \& Poch-Olivé, M. L. (2007). Formatos interactivos y funciones ejecutivas en el desarrollo temprano. Revista de Neurología, 44(2), 61-65. Recuperado de https://medes.com/publication/28141

Schoemaker, K., Bunte, T., Wiebe, S. A., Espy, K. A., Deković, M., \& Matthys, W. (2012) Executive function deficits in preschool children with ADHD and DBD. Journal of Child Psychology and Psychiatry, 53(2), 111-119. doi: 10.1111/j.14697610.2011.02468.x

Semrud-Clikeman, M., Walkowiak, J., Wilkinson, A., \& Butcher, B. (2010). Executive functioning in children with Asperger syndrome, ADHD-combined type, ADHDpredominately inattentive type, and controls. Journal of Autism and Developmental Disorders, 40(8), 1017-1027. doi: 10.1007/s10803-010-0951-9

Servera-Barceló, M. (2005). Modelo de autorregulación de Barkley aplicado al Trastorno por déficit de atención con hiperactividad: una revisión. Revista de Neurología, 40(6), 358-368. Recuperado de Researchgate

Soprano, A. M. (2003). Evaluación de las funciones ejecutivas en el niño. Revista de Neurología, 37(1), 44-50. Recuperado de http://www.neurologia.com/pdf/ Web/3701/p010044.pdf

Spencer, T. J. (2005). ADHD and comorbidity in childhood. The Journal of Clinical Psychiatry, 67, 27-31. Recuperado de http://www.ncbi.nlm.nih.gov/pubmed/16961427

Valdizán, J. R., Mercado, E., \& Mercado-Undanivia, A. (2007). Características y variabilidad clínica del Trastorno por déficit de atención/hiperactividad en niñas. Revista de Neurología, 44(2), 27-30. Recuperado de https://medes.com/publication/28134

Van De Voorde, S., Roeyers, H., Verté, S., \& Wiersema, J. R. (2010). Working memory, response inhibition, and within-subject variability in children with Attention deficit/hyperactivity disorder or reading disorder. Journal Clinical and Experimental Neuropsychology, 32, 366-379. doi: 10.1080/13803390903066865

Vélez-van-Meerbeke, A., Zamora, I. P., Guzmán, G., Figueroa, B., Cabra, C. L., \& TaleroGutiérrez, C. (2013). Evaluación de la función ejecutiva en una población escolar con síntomas de déficit de atención e hiperactividad. Neurología, 28(6), 348-355. doi: 10.1016/j.nrl.2012.06.011

Wechser, D. W. (2010). Wisc-IV: Test de inteligencia para niños. Buenos Aires: Paidós. Wicks-Nelson, R., Israel, A. C., \& Frías, C. F. (1997). Psicopatología del Niño y del Adolescente. ( ${ }^{\mathrm{a}}$ Ed.). Madrid: Prentice Hall. http://www.casadellibro.com/libro-psicopatologia-del-nino-y-del-adolescente/9788483220153/571170

Willcut, E. G., Doyle, A. E., Nigg, J. T., Faraone, S. V., \& Pennington, B. F. (2005). Validity of the executive fintion theory of Attention-Deficit/Hiperactivity Disorder: A meta-analytic review. Biological Psychiatry, 57, 1336-1346. doi:10.1016/j.biopsych.2005.02.006

Willcutt, E. G. (2012). The prevalence of DSM-IV Attention-Deficit/Hyperactivity Disorder: a meta-analytic review. Neurotherapeutics, 9(3), 490-499. doi: 10.1007/ s13311-012-0135-8

Zelazo, P. D., Carter, A., Reznick, J. S., \& Frye, D. (1997). Early development of executive function: a problem-solving framework. Review of General Psychology, 1,198226. doi: $10.1037 / 1089-2680.1 \cdot 2.198$ 